\title{
QUORUM SENSING ANALYSIS AND EFFECT OF BACTERIOCIN IN CONTROLLING THE BIOFILM FORMATION OF PSEUDOMONAS AERUGINOSA
}

\section{S. PRIYA DHARSINI ${ }^{1}$, G. BHUVANESHWARI ${ }^{2}$, M. KALYANI ${ }^{3}$, ROSY VENILLA ${ }^{4}$}

${ }^{1}$ Department of Microbiology, Saveetha Medical College, ${ }^{2}$ Department of Microbiology, Saveetha Medical College, ${ }^{3} \mathrm{Head}$ of the Department, Panimalar Medical College, ${ }^{4} \mathrm{Head}$ of the Department, Saveetha Medical College

Email: prickipridarshi@gmail.com

Received: 10 Jul 2020, Revised and Accepted: 08 Sep 2020

ABSTRACT

Objective: Pseudomonas aeruginosa is the commonest causative agent of Hospital Acquired Infection (HAI). Resistance of Pseudomonas aeruginosa towards disinfectants and antibiotics is high when compared to other organisms. There are various reviews on antibacterial effect of herbal extracts and nanoparticles against Pseudomonas aeruginosa. Thus, this study aims to prove the effectiveness of bacteriocin against Pseudomonas aeruginosa, which may help in benefiting the health care center by replacing normal disinfectants.

Methods: 100 strains of Pseudomonas aeruginosa were collected from different patients, with a study period of 6 mo. Ammonium sulfate precipitation method was used to extract bacteriocin and its efficiency was checked by paper disc diffusion assay. Biofilm formation assay and quorum sensing analysis was performed by Microtitre plate methods and Thin Layer Chromatography (TLC), respectively.

Results: In this study, 91\% of P. aeruginosa strains were strong, 8\% were intermediate, and 1\% were weak biofilm producers. From TLC analysis, $67 \%$ of the strains produced Acyl Homoserine Lactone molecules. Out of which, $49 \%$ has shown unknown analytes of Retardation factor (Rf) value greater than 1. The Rf values identified were 3 unsubstitutedC4 (5\%), 3 unsubstituted C6 (4\%), 3 oxo C8 (3\%), 3 oxo C4 (3\%), 3 oxo C6 (2\%), 3 oxo C1 (1\%). Biofilm production, before and after bacteriocin exposure, was proved significant by paired t-test.

Conclusion: Quorum sensing was confirmed to play a major role in biofilm formation. As bacteriocin was found to be effective in controlling the biofilm formation, it can be incorporated in any disinfectant, which helps in controlling the transmission of infection caused by Pseudomonas aeruginosa.

Keywords: Bacteriocin, Biofilm formation, Pseudomonas aeruginosa

(C) 2020 The Authors. Published by Innovare Academic Sciences Pvt Ltd. This is an open access article under the CC BY license (http://creativecommons.org/licenses/by/4.0/) DOI: http://dx.doi.org/10.22159/ijcpr.2020v12i6.40283. Journal homepage: https://innovareacademics.in/journals/index.php/ijcpr

\section{INTRODUCTION}

Pseudomonas aeruginosa is a non-fermentative, aerobic, gram negative rod that normally lives in moist environments. It has minimal nutritional requirements while being able to use several organic compounds. Occasionally, Pseudomonas aeruginosa can colonize moist areas of human body sites such as the perineum, axilla, ear, nasal mucosa and throat. The higher colonization rates can be encountered by the patient following treatment with broad spectrum antibiotics [1]. Colonization is common in respiratory tract of ventilated patients, gastro intestinal tract of patients receiving anti-cancer chemotherapy, and on the skin of burn patients [2]. Pseudomonas aeruginosa is typically an opportunistic pathogen that seldom causes disease in healthy subjects. Normally it causes infection by disturbing the physical barriers (skin or mucous membranes) [2]. Pseudomonas aeruginosa is generally a nosocomial pathogen in accordance to data collected from the centre for Disease Control and prevention National nosocomial infection surveillance system in the USA. Pseudomonas aeruginosa is the second most common cause of nosocomial pneumonia, the third most common cause of nosocomial urinary tract infections, and $7^{\text {th }}$ most cause of nosocomial bacteremia. Pseudomonas aeruginosa sometimes causes life-threatening disease due to its resistance towards antibiotics [3]. Pseudomonas aeruginosa may also cause "Hot tub Rash" (Dermatitis) and swimmer's ear. Pseudomonas produce many toxins such as Non-diffusible toxins: Exotoxins $\mathrm{S}, \mathrm{U}, \mathrm{T}$ and $\mathrm{Y}$ and diffusible toxins such as protease, phospholipase, hemolysins, elastase, pyocyanin. The organism also produces various pigments such as pyocyanin (green pigment), Flourescein (pyoverdin/yellow pigment), pyorubin (imparted red colour), pyomelanin (imparted brown colour). There is always an association between a pathogenic organism and biofilm formation.

Pathogenesis and biofilm formation of the organism is always directly proportional to quorum sensing. Biofilms are defined as microbially derived sessile communities characterized by cells that are irreversibly attached to substratum or each other. They are embedded in a matrix of polymerized substances (EPS) and exhibit an altered phenotype with respect to growth and gene transcription. Within a biofilm, bacteria communicate with each other by the production of chemostatic particles. Microorganism growing in a biofilm is intrinsically resistant to antimicrobial agents than planktonic cells. Biofilms are associated with many medical conditions, including indwelling medical devices, dental plaques, upper respiratory tract infections and urogenital infections.

Quorum sensing is a cell to cell signaling and it is the ability to detect and to respond to cell population density by gene regulation. Pseudomonas aeruginosa uses quorum sensing to coordinate the formation of biofilm, swarming motility, exopolysaccharide production, virulence and cell aggregation. These bacteria can grow within a host without harming until reaching a threshold concentration. They become aggressive and multiply in numbers sufficient to overcome host's immune system, and forming a biofilm leading to the formation of disease. Quorum sensing uses autoinducers mainly Acyl Homoserine Lactone (AHL) as signaling molecules and can be found out by using techniques such as Thin Layer Chromatography (TLC), High-Performance Liquid Chromatography (HPLC), beta-galactosidase enzyme and electrophoresis. At least two complete quorum-sensing systems, las and rhl, are present in the opportunistic human pathogen, Pseudomonas aeruginosa. These systems are known to control the expression of a number of virulence genes in response to bacterial cell density. Moreover, Pseudomonas aeruginosa is resistant to disinfectants and this has been contributed to easier spread of the organism in a tertiary care centre. Pyocins are produced by more than $90 \%$ of Pseudomonas aeruginosa strains and each strain may synthesis several pyocins, where the pyocin genes are situated on $P$. 
aeruginosa chromosomes. They are proteinatious particles which can be directed against their own species and such pyocins also have antibiofilm activity. In our study, bacteriocin has been tested for efficiency in order to support disinfectants in removing the biofilms and provide a good sanitary condition in a tertiary care centre.

\section{MATERIALS AND METHODS}

\section{Study period}

The cross-sectional study was performed for a period of 6 mo from January 2018 to June 2018, at Saveetha Medical College and Hospital, located at Thandalam, Kancheepuram. The samples were received from in-patients and outpatients from various departments to the Clinical Microbiology Laboratory of Saveetha Hospital.

\section{Sample size}

Out of 4728 samples, 100 strains of Pseudomonas aeruginosa were isolated from Clinical Microbiology Laboratory and were collected and sub-cultured.

\section{Inclusion criteria}

Pseudomonas aeruginosa isolates from various samples like urine, blood, Respiratory samples such as pleural fluid, sputum, broncheo alveolar larvage, Endotracheal swabs and exudates such as CSF, wound swab, tissue, pus,ascetic fluid etc., were included in this study.

\section{Exclusion criteria}

Samples having strain other than pseudomonas aeruginosa was excluded from the study.

\section{Sample collection and transport}

All the samples were received within two hours of collection from the patients. The urine, respiratory and exudate samples were received in a sterile, screw-capped wide mouthed, leak proof containers. Blood samples were received in biphasic brain heart infusion broth. In case of urine, the samples from suspected patients were collected by standard sterile techniques and patients were instructed to collect mid-stream clear, catch urine. In case of catheterized patients, aspiration was done from port of the urinary catheter, using sterile syringes. The collected samples were processed immediately without any delay. In case of delay the samples were refrigerated at $4^{\circ} \mathrm{C}$.

\section{Bacteriocin extraction-ammonium sulfate precipitation method}

After optimization, Ps. aeruginosa SA188 was grown in BHI at $29{ }^{\circ} \mathrm{C}$ for $18 \mathrm{~h}$ and the cells were separated by centrifugation at $6000 \mathrm{Xg}$ for $30 \mathrm{~min}$ at $4{ }^{\circ} \mathrm{C}$.

The cell-free supernatant (CFS) was adjusted to $\mathrm{pH} 7.0$ and was filter sterilized by a $0.45 \mu \mathrm{m}$ pore size membrane filter. This crude bacteriocin preparation was partially purified by $70 \%$ Ammonium sulfate precipitation at $4{ }^{\circ} \mathrm{C}$. The precipitate was sedimented by centrifugation at $6000 \mathrm{X} \mathrm{g}$ for $45 \mathrm{~min}$ at $4{ }^{\circ} \mathrm{C}$. The resulting pellet was suspended in 50 mmol Sodium Phosphate Buffer of $\mathrm{pH} 7.0$ and referred as partially purified bacteriocin preparation [4]. The activity units (AU) of both crude and the partially purified bacteriocin preparations were determined by paper disc diffusion assay.

\section{Bacteriocin efficiency-paper disc diffusion assay}

Paper disc assay was done in Muller Hinton agar.

\section{Biofilm formation analysis-MTCP method}

\section{Growing a biofilm}

Grow a culture of the wild-type Pseudomonas aeruginosa or mutant strain overnight in a rich medium (i.e. LB). Pseudomonas aeruginosa with and without bacteriocin exposure was incubated in the plate. Dilute the overnight culture 1:100 into fresh medium for biofilm assays. A standard biofilm assay medium for $P$. aeruginosa is Brain Heart Infusion Broth. Add $100 \mu \mathrm{l}$ of the dilution per well in a 96 well dish. For quantitative assays, we typically use 4-8 replicate wells for each treatment. Incubate the microtiter plate for $4-24 \mathrm{~h}$ at $37^{\circ} \mathrm{C}$.

\section{Staining the biofilm}

After incubation, dump out cells by turning the plate over and shaking out the liquid.

Gently submerge the plate in a small tub of water (i.e., use the bottoms of pipette tip boxes for P1000 pipetmen as the tub). Shake out water. Repeat this process a second time. This step helps remove unattached cells and media components that can be stained in the next step, and significantly lowers background staining. Add $125 \mu \mathrm{l}$ of a $0.1 \%$ solution of crystal violet in water to each well of the microtiter plate. Wear gloves and a lab coat while making the solution. Use caution when weighing out the $\mathrm{CV}$ as the powder is hygroscopic and readily stains clothing, skin, etc. Incubate the microtiter plate at room temperature for 10-15 min. Rinse the plate 3-4 times with water by submerging in a tub of water as outlined above, shake out and blot vigorously on a stack of paper towels to rid the plate of all excess cells and dye. Turn the microtiter plate upside down and dry for a few hours or overnight. For qualitative assays, the wells can be photographed when dry.

\section{Quantifying the biofilm}

Add $125 \mu \mathrm{l}$ of $30 \%$ acetic acid in water to each well of the microtiter plate to solubilize the CV. Incubate the microtiter plate at room temperature for 10-15 min. Transfer $125 \mu \mathrm{l}$ of the solubilized CV to a new flat bottomed microtiter dish. Quantify absorbance in a plate reader at $550 \mathrm{~nm}$ using $30 \%$ acetic acid in water as the blank [5].

\section{Grading the biofilm}

The OD values were calculated for all tested strains and negative controls [6]. The cut-off value (ODc) was established. It is defined as three standard deviations (SD) above the mean OD of the negative control+(3* SD of the negative control). Final OD value of a tested strain was expressed as average OD value of the strain reduced by average $O D c$ value $(O D=$ average $O D$ of a strain-oDc); $O D c$ value was calculated for each microtitre plate separately. When a negative value was obtained it was presented as zero, while any positive value indicated biofilm production. For easier interpretation of the results strains was categorized into the following categories:

Non biofilm producer (0) OD $<$ ODc

Weak biofilm producer $(+\mathrm{OR} 1)=\mathrm{ODc}<\mathrm{OD}<2 * \mathrm{ODc}$

Moderate biofilm producer $(++\mathrm{OR} 2)=\mathrm{ODc} * 2<\mathrm{OD}<4 * \mathrm{ODc}$

Strong biofilm producer $(+++\mathrm{OR} 3)=4^{*} \mathrm{OD} c<\mathrm{OD}$

\section{Statistical analysis:}

Statistically significant variance $\left({ }^{*} \mathrm{p}<0.05\right)$ for collected data was determined by two-paired (tailed) ANOVA test. The $t$ value was1.9685 and $p$-value was 0.0502 . Therefore $p$-value was found to be insignificant and pyocin was found to be effective

\section{Quoroum sensing analysis-thin layer chromatography}

Extracts for TLC were prepared from $100 \mathrm{ml}$ cultures after growth in LB medium for $20 \mathrm{~h}$ at $25^{\circ} \mathrm{C}$ with agitation of $150 \mathrm{r}$. p. m. Bacteria were removed by centrifugation, the supernatants were extracted twice with equal volumes of ethyl acetate acidi fied with $0.5 \%$ of formic acid, and the combined extracts were dried,filtered, and evaporated to dryness. Cultures extract were dissolved in 400-600 ll of HPLC-grade ethyl acetate. Synthetic AHLs or extract samples dissolved in ethyl acetate, in volumes of 10-20 microlitres were spotted onto c18 reversed-phase TLC plates and the chromatogram was developed using a solvent system of methanol/water $(60: 40, \mathrm{v} / \mathrm{v})$. A small amount of the mixture to be analyzed is spotted near the bottom of this plate. Then Rf value was calculated and the AHL molecules were graded accordingly.

\section{RESULTS}

The prospective study was conducted at Clinical Microbiology Laboratory of Saveetha Medical College and Hospital during the period of mid of December 2018 to mid of April 2019. Ethical 
clearance was obtained. Ethical clearance number is SMC/IEC/2018/11/261. Out of 100 samples received, $66 \%$ samples

were from male and $34 \%$ samples were from female.

Table 1: Chromatographic properties of $N$-acyl-HSLs

\begin{tabular}{|c|c|}
\hline $3-\mathrm{Oxo}-\mathrm{C}_{4}$ & 0.82 \\
\hline $3-0 \times 0-\mathrm{C}_{6}$ & 0.68 \\
\hline $3-\mathrm{Oxo}-\mathrm{C}_{8}$ & 0.41 \\
\hline $3-0 \times 0-\mathrm{C}_{10}$ & 0.18 \\
\hline 3-Oxo- $\mathrm{C}_{12}$ & 0.07 \\
\hline 3-Unsubstituted $\mathrm{C}_{4}$ & 0.77 \\
\hline 3-Unsubstituted $\mathrm{C}_{8}$ & 0.23 \\
\hline 3-Unsubstituted $\mathrm{C}_{10}$ & 0.09 \\
\hline 3-Unsubstituted $\mathrm{C}_{12}$ & 0.02 \\
\hline
\end{tabular}

\section{Bacteriocin extraction}

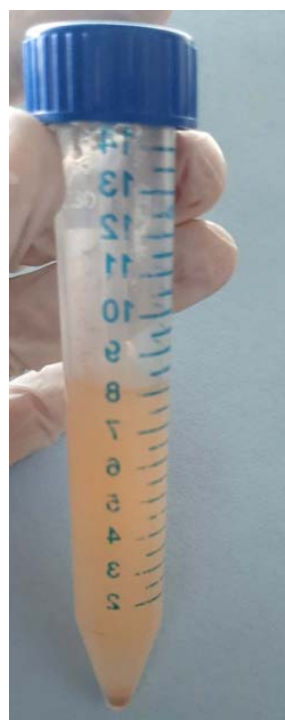

Fig. 1: Extracted bacteriocin

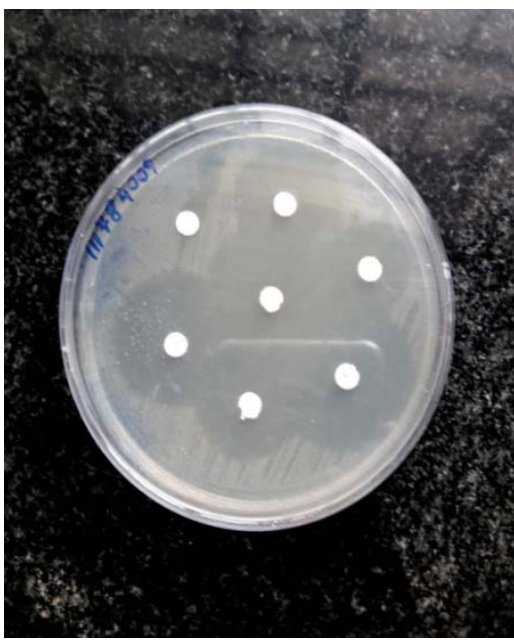

Fig. 2: PPDA

The extracted bacteriocin was found to be efficient by means of Paper Disc Diffusion Assay. The bacteriocin was found to control biofilm formation both qualitatively and quantitatively which was proved by statistical analysis.

\section{Biofilm formation}

Out of 100 pathogenic strains obtained from 100 samples, $91 \%$ strains showed strong biofilm formation; 8\% strains showed intermediate biofilm formation; $1 \%$ strain showed weak biofilm formation; there were no non-biofilm formers according to our study.

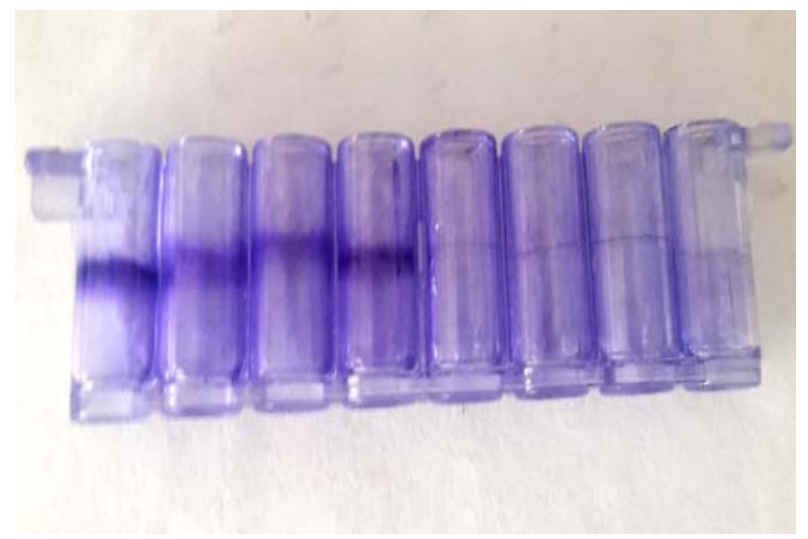

Fig. 3: Biofilm formation without and with bacteriocin

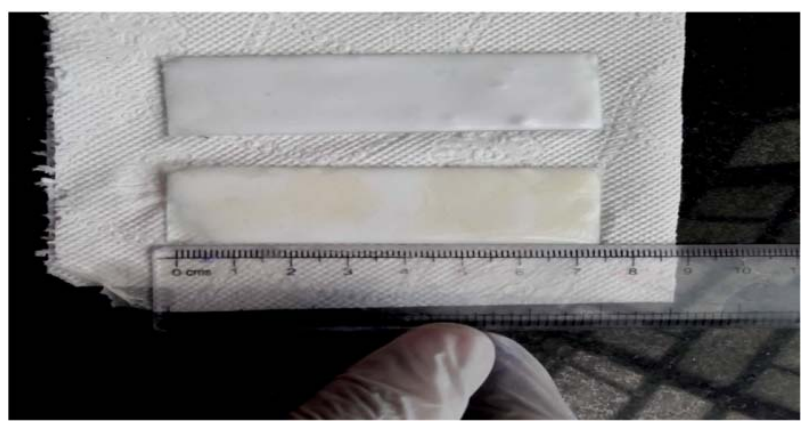

Fig. 4: Measuring the Rf value

\section{Thin layer chromatography}

In our study, different AHL has been classified. $67 \%$ of the strains produce Acyl Homoserine Lactone (AHL) molecules. Out of which, $49 \%$ has shown unknown analyte of retardation value greater than 1. The $\mathrm{Rf}$ value identified were 3 unsubstituted $\mathrm{C} 4(5 \%), 3$ unsubstituted C6 (4\%), 3 oxo C8 (3\%), 3 oxo C4 (3\%), 3 oxo C6 (2\%) and 3 oxo $\mathrm{C} 1(1 \%)$

\section{DISCUSSION}

In this present study, 100 samples were collected from patients infected with Pseudomonas aeruginosa. The samples were collected from the period of mid of December 2018 to April 2019. The samples received to Clinical Microbiology Laboratory of Saveetha Medical College and Hospital was included. Out of 100 samples, 66\% were from male gender and $34 \%$ were from female gender.

Among 100 samples that were included in this study, 39\% sample was wound swab, $29 \%$ of the sample was urine, $12 \%$ of the sample was sputum, $5 \%$ of the sample was from pus, $4 \%$ of the sample was 
from blood and the rest of the samples were ear swab (3\%), bronchial wash (1\%) and finally endo-tracheal sample (1\%).

In this study, $25 \%$ of the samples were from patients of age group $41-60$, followed by $25 \%$ of the samples were from patients of age group above 60 and $21 \%$ of the samples were from patients of age group $21-40$ and $14 \%$ of the samples were from patients of age group 0-20.

The pseudomonas aeruginosa strains collected for this study showed resistance to Cefepime (19\%), followed by Ceftazidime (28\%), Ciprofloxacin (26\%), Meropenem (25\%), Imipenem (26\%), Gentamicin (22\%), Co-trimaxazole (16\%) and Piperacillintazobactam (21\%). In a study conducted by [7], there was similar corelation of antibiotic resistant pattern of Pseudomonas aeruginosa which showed a slightly higher resistance to drugs, Cefefpime $(36.27 \%)$, followed by Ceftazidime (35.30\%), Ciprofloxacin (31.37\%), Gentamicin (28.43\%), Meropenem (20.59\%) and Levofloxacin (32.35\%). Bacteriocins are antibiotic substances produced by many species of bacteria which are thought to be inhibitory for strains of the same or closely related species. Bacteriocins are protein in nature and range from simple proteins to particles resembling bacteriophage components. Bacteriocinogenic strains of P. aeruginosa produce several types of bacteriocins. The synthesis of these bacteriocins can be increased by mitomycin $C$ treatment. In a study [8], pyocins were able to kill Neisseria gonnorhoeae, Neisseria meningitis, Neisseria lactamica

In a study conducted [9], $\mathrm{N}$-acyl homoserine lactones produced by $\mathrm{P}$. fluorescens 395 were idenfied to be C4 -HSL and 30C8-HSL. The protease gene in Puorescens is regulated by the AHL -based quorum sensing system at a transcriptional level during the late exponential growth phase.

In a study conducted [10] mixture of OHHL, HHL, OHL and BHL (AHL) molecules were detected by thin layer chromatography.

In a study conducted [11], production of several AHLs (C6-AHL, oxoC6AHL, C8-AHL, C10-AHL, and C12-AHL) by positive clones was confirmed by Mass Spectrometry.

In a study conducted [12], N-(3-Oxooctanoyl)-L-homoserine lactone and $\mathrm{N}$-(3-oxohexanoyl)-L-homoserine lactone were the most abundant AHLs produced by recombinant YspI in E. coli and by nonrecombinant wild-type Y. pestis. Therefore, 3-oxo-C6-ACP and 3oxo-C8-ACP are the primary substrates for YspII.

Some Vibrionaceae species have been reported to produce several kinds of AHLs, such as L. anguillarum (C6-HSL, 3-hydroxy-C6-HSL and 3-oxo-C10-HSL), Afischeri (3 -oxo-C6-HSL and C8-HSL), V. harveyi (3-hydroxyC4-HSL), V. vulnficus (C4 -HSL, 3-oxo-C10-HSL 3-oxo-C12-HSL, and minor amounts of C6-HSL, 3-oxo-C10-HSL and 3-oxo-C14-HSL) and V. salmonicida (3-oxo-C6-HSL and C6-HSL). Molecules such as C6, 30C10, 30C12, and 30C14 AHLs, and were found only in a small percentage of the bacterial population $[13,14]$.

Microtitre tissue culture plate method was chosen to perform biofilm formation assay. Tube method and Cong red agar method was excluded from the study because Afreenish Hassan et al. did a comparative study on biofilm formation and found that out of three methods, microtitre tissue culture plate method was the best method and a sensitive method to carry biofilm formation [15]. Statistically significant variance $\left({ }^{*} \mathrm{p}<0.05\right)$ for collected data was determined by two-paired (tailed) ANOVA test. The t value was1.9685 and p-value was 0.0502 . Therefore $p$-value was found to be insignificant and pyocin was found to be effective. Pyocin can inhibit biofilm formation and was proved to have anti-bacterial activity. In various studies, Pseudomonas aeruginosa has shown to be resistant against chlorohexidine [16], glutaraldehyde, sodium hypochloride, phenolics, Quartenary ammonium compounds (QAC) [17], hexachlorophene [18] and didecyldimethyl ammonium chloride [19]. Phospholipids, fatty andamp; neutral lipids in the cell wall of $P$. aeruginosa makes it more resistant to benzalkonuium chloride [20]. Cavicide, cidexplus, Clorox, export, Lysol, wavicide, renalin, formaldehyde, para acetic acid, hydrogen peroxide, phenol and cupric ascorbate [21]. But chlorine dioxide has been shown to be effective against multi drug resistant $P$. aeruginosa [22]. So,
Bacteriocins especially the Pyocins can be used to support the disinfectants. Bacteriocins can not only support disinfectants; they can also support antibiotics when extracted along with mitomycin C. So, further studies can be in the area of using bacteriocins to support antibiotics in vivo because of the emergence of multi drug resistant and extreme drug resistant Pseudomonas aeruginosa.

Along with TLC, better molecular methods for detecting quorum sensing signals like beta galactosidose assay, electrophoresis, HPLC will be used as a continuation of the present study in the future. The present study used the crude extract of bacteriocin. Based on this study the exact molecule responsible for antibacterial activity will be decided and purified using column chromatography.

\section{CONCLUSION}

There is a strong association with quorum sensing, biofilm formation and pathogenesis of Pseudomonas aeruginosa. Disinfectants have been ineffective in controlling the nosocomial Spread of Pseudomonas aeruginosa. So, Bacteriocins especially the Pyocins can be used to support the disinfectants. Bacteriocins can not only support disinfectants; they can also support antibiotics when extracted along with mitomycin C. So, further studies can be in the area of using bacteriocins to support antibiotics in vivo because of the emergence of multi drug resistant and extreme drug resistant Pseudomonas aeruginosa. There are still some quorum sensing signals which are not indexed and are therefore considered as unidentified or unknown analytes. So, further studies needed for identifying new quorum sensing signals. Along with TLC, better molecular methods for detecting quorum sensing signals like beta galactosidose assay, electrophoresis, HPLC will be used as a continuation of the present study in the future. The present study used the crude extract of bacteriocin. Based on this study the exact molecule responsible for antibacterial activity will be decided and purified using column chromatography.

\section{ACKNOWLEDGEMENT}

I would like to thank Saveetha Medical College for giving me a wonderful opportunity to carry out my thesis. I would like to thank my guide, Dr. G. Bhuvaneshwari to encourage me during my full course of bench work. I would also like to thank Dr. M. Kalyani, who constantly went on determining me in completion of my thesis work. I also like to thank my other faculty members and friends. Last but not the least, I would like to thank my parents who played a very great part in all of my process.

\section{FUNDING}

Nil

\section{AUTHORS CONTRIBUTIONS}

All the authors have contributed equally.

\section{CONFLICT OF INTERESTS}

\section{Declared none}

\section{REFERENCES}

1. Waheed Ullah, Muhammad Qasim, Hazir Rahman, Yan Jie, Noor Muhammad. Beta-lacatmase-producing pseudomonas aeruginosa: phenotypic characteristics and molecular identification of virulence genes. J Chinese Med Association 2017;80:173-7.

2. GM Rossolini, E Mantengoli. Treatment and control of severe infections caused by multiresistant pseudomonas aeruginosa. Clin Microbiol Infect 2005;11(Suppl 4):17-32.

3. Kuan Yin Lina, Tsai Ling Lauderdaleb, Jann Tay Wanga B, Shan Chwen Chang. Carbapenem-resistant pseudomonas aeruginosa in taiwan: prevalence, risk factors, and impact on outcome of infections. J Microbiol Immunol Infection 2016;49:52-9.

4. Sehar Afshan Naz, Sheikh Ajaz Rasool. Isolation, production and characterization of bacteriocins produced by strains from indigenous environments. Pak J Bot 2013;45:261-7.

5. O'Toole GA. Microtiter dish biofilm formation assay. JoVE 47; 2011. 
6. Tayal Ruchi, Baveja Sujata, De Anuradha. Comparison of phenotypic methods for the detection of biofilm production in uro-pathogens in a tertiary care hospital in India. Int J Curr Microbiol Appl Sci 2015;4:840-9.

7. Piyush Tripathi, Gopa Banerjee, Shivani Saxena, Mahendra Kumar Gupta, PW Ramteke. Antibiotic resistance pattern of pseudomonas aeruginosa isolated from patients of lower respiratory tract infection. Afr J Microbiol Res 2015;5:2955-9.

8. Stephen A Morse, Patrick Vaughan, Deanne Johnson, Barbara $\mathrm{H}$ Iglewski. Inhibition of Neisseria gonorrhoeae by a bacteriocin from pseudomonas aeruginosa. Am Soc Microbiol 1976;1:354-62.

9. $\mathrm{M}$ Liu, $\mathrm{H}$ Wang, MW Grffths. Regulation of alkaline metalloprotease promoter by $\mathrm{N}$-acyl homoserine lactone quorum sensing in pseudomonas fluorescens. J Appl Microbiol 2007;103:2174-84.

10. Ahmad Humayan Kabir, Anindya Ghosh Roy, Mohammad Firoz Alam, Rafiul. Detection of quorum sensing signals in gramnegative bacteria by using reporter strain CV026. Not Sci Biol 2010;2:72-5.

11. Tomohiro Kawaguchi, Yung Pin Chen, R Sean Norman, Alan W Decho. Rapid screening of quorum-sensing signal n-acyl homoserine lactones by an in vitro cell-free assay. Appl Environ Microbiol 2008;74:3667-1.

12. J Paul Kirwan, Ty A Gould, Herbert P Schweizer, Scott W Bearden, Robert C Murphy, Mair EA Churchill. Quorum-sensing signal synthesis by the yersinia pestis acyl-homoserine lactone synthase yspi. J Bacteriol 2006;188:784-8.

13. Q Yang, Y Han, XH Zhang. Detection of quorum sensing signal molecules in the family vibrionaceae. J Appl Microbiol 2011;110:1438-48.

14. Fiorela Nievas, Pablo Bogino, Fernando Sorroche, Walter Giordano. Detection, characterization, and biological effect of quorum-sensing signaling molecules in peanut-nodulating bradyrhizobia. Sensors 2012;12:2851-73.

15. Hassan A, Usman J, Kaleem F, Omair M, Khalid A, Iqbal M. Evaluation of different detection methods of biofilm formation in the clinical isolates. Braz J Infect Dis 2011;15:305-11.

16. Thomas, Louise. Development of resistance to chlorhexidine diacetate in Pseudomonas aeruginosa and the effect of a 'residual' concentration. J Hospital Infection 2000;46:297-303.

17. Romao, Celia Maria Carvalho Pereira Araujo, Faria, Yaisa Naziozeno de, Pereira, Luciana Roberto, et al. Susceptibility of clinical isolates of multiresistant Pseudomonas aeruginosa to a hospital disinfectant and molecular typing. Memorias Instituto Oswaldo Cruz 2005;100:541-8.

18. Sutton L, Jacoby GA. Plasmid-determined resistance to hexachlorophene in pseudomonas aeruginosa. Antimicrob Agents Chemother 1978;13:634-6.

19. Marwan Abdallah, Corinne Benoliel, Pauline Ferreira Theret, Djamel Drider, Pascal Dhulster, Nour Eddine Chihib. Effect of culture conditions on the resistance of Pseudomonas aeruginosa biofilms to disinfecting agents. Biofouling 2015;31:49-59.

20. Y Sakagami, H Yokoyama, H Nishimura, Y Ose, T Tashima. Mechanism of resistance to benzalkonium chloride by Pseudomonas aeruginosa. Appl Environ Microbiol 1989;55:2036-40.

21. Sagripanti, Jose Luis, Bonifacino, Aylin. Resistance of Pseudomonas aeruginosa to liquid disinfectants on contaminated surfaces before formation of biofilms: J AOAC Int 2000;83:1415-22.

22. Hinenoya A, Awasthi SP, Yasuda N. Chlorine dioxide is a better disinfectant than sodium hypochlorite against multi-drug resistant staphylococcus aureus, pseudomonas aeruginosa, and acinetobacter baumannii. Japan J Infect Dis 2015;68:276-9. 